SHS Web of Conferences 21, 02001 (2015)

DOI: $10.1051 /$ shsconf/ 20152102001

(C) Owned by the authors, published by EDP Sciences, 2015

\title{
Science et débat public sur le vivant
}

\author{
Florence Piron ${ }^{1}$
}

Département d'information et de communication, Université Laval, Québec (Qc) G1V 0A6 Canada et Association science et bien commun

\begin{abstract}
Résumé. Quels sont les repères éthiques et politiques que les sociétés se donnent pour respecter la vie et le vivant selon leur culture? D'où viennent les débats et les dissensus? Au-delà des débats de société suscités par le vivant aujourd'hui, il est essentiel de mettre au jour les présupposés épistémologiques qui, en plus des politiques scientifiques, orientent les recherches sur le vivant qui, ensuite, sont mobilisées comme moyen de légitimation des arguments dans ces débats. La science apparaît alors comme une institution non neutre, inscrite dans la culture, qui influence les débats publics sur le vivant, au lieu de seulement les éclairer.
\end{abstract}

\begin{abstract}
According to its culture, what are society's ethical and political guidelines regarding the respect of life? Where do debates and disagreements come from? Beyond the social debates raised by these issues, it is essential to uncover, in addition to scientific policies, the underlying epistemologies that guide research in life sciences and are then mobilized as legitimate means of argument in these debates. Science appears then as a non-neutral, embedded in a culture, influencing public debates instead of just informing.
\end{abstract}

\section{Introduction}

Que serait un débat public démocratique idéal sur le vivant et ses différentes déclinaisons? Si on considère que la démocratie doit permettre à tous les citoyens et citoyennes de faire entendre leur voix dans les décisions qui engagent toute leur collectivité, que ce soit par le moyen du vote ou de la participation citoyenne et publique, un débat public sur le vivant qui soit véritablement démocratique permettrait que les valeurs des différentes composantes de la société soient entendues et fassent l'objet d'une écoute similaire de la part des décideurs. Or, dans bien des pays, les citoyens et citoyennes qui, par exemple, expriment des doutes au sujet des OGM, des vaccins ou des cellules souches sont vite tournés en ridicule par les médias et les associations scientifiques qui les jugent conservateurs, craintifs et fermés au progrès. Quand ce sont des scientifiques qui expriment de tels doutes, ils sont ostracisés, comme l'a vécu le biologiste Gilles-Éric Séralini qui travaille sur les OGM [1]. En fait, les chercheurs et chercheuses prennent rarement le temps d'écouter et de discuter avec les non-scientifiques qui s'inquiètent des conséquences des recherches sur le vivant: leur intervention dans le débat public consiste davantage à faire des conférences grand public ou de la vulgarisation dans le but de convaincre et rassurer la population qu'à écouter.

${ }^{1}$ Auteure de correspondance Florence.Piron@com.ulaval.ca

This is an Open Access article distributed under the terms of the Creative Commons Attribution License 4.0, which permits unrestricted use, distribution, and reproduction in any medium, provided the original work is properly cited. Article available at 5 tip://www.shs-conterences.org or $n$ ittp://dx.dol.org/10.1051/shscont/20152102001 
La bioéthique nous amène collectivement à réfléchir aux effets des technologies du vivant sur la société. Mais il faut se tourner vers l'épistémologie sociale et politique [2] pour comprendre ce qui, de manière interne à la science contemporaine, a rendu pour les scientifiques si difficile de participer de manière simple, équitable et démocratique, aux débats publics sur la science du vivant, sans, par exemple, user d'argument d'autorité scientifique ou de condescendance envers les non-scientifiques. C'est ce que cet article propose de faire à travers plusieurs exemples ${ }^{2}$.

\section{Celles qui savent et celles qui ne savent pas}

« Vous ne pouvez pas faire griller davantage le mil, même si vous trouvez que les bébés le digèrent mieux, car vous allez détruire les vitamines! »

Cette phrase, les femmes analphabètes d'un quartier de Ouagadougou qui travaillaient en 1992 dans une petite coopérative de farine de transition pour bébés (installée dans un quartier où sévissait la malnutrition infantile) l'ont entendue de la bouche d'une des infirmières du dispensaire qui leur avait montré comment fabriquer cette farine. Que s'était-il passé? Après leur retour de cette formation, les femmes de la coopérative n'étaient pas satisfaites de la farine qu'elles avaient appris à produire. Elles avaient donc décidé de modifier la recette jusqu'à obtenir une plus grande qualité en faisant griller davantage les composants de la farine. Fières de leur succès, elles étaient allées raconter leur histoire aux infirmières, pour partager leur nouveau savoir. Loin de rencontrer une oreille bienveillante, elles reçurent la réponse ci-dessus et en furent bien dépitées, car elles ignoraient ce qu'étaient des vitamines... Cette histoire, recueillie il y a très longtemps dans le cadre d'une enquête sur la place des savoirs des femmes dans trois projets de développement international au Sahel [3], est pour moi emblématique des rapports de pouvoir qui peuvent s'installer entre des savoirs, pourtant tous nécessaires à la compréhension de la santé.

D'une part, il y avait ces femmes analphabètes ou très peu instruites qui tentaient de contribuer au mieux-être des enfants de leur quartier, incluant leurs propres enfants. Observatrices, intelligentes, elles ont utilisé une méthode empirique (essai-erreur) pour innover en améliorant un produit qui leur paraissait insatisfaisant. Elles ont produit un savoir extrêmement local, expérientiel, car directement tiré de leurs observations des bébés nourris avec leur farine. Peut-être leur nouvelle recette n'expliquait-elle pas le mieux-être des enfants et la meilleure apparence de la farine; peut-être était-ce un hasard, une contingence, le résultat d'un autre facteur ou la correction d'une erreur. Nous ne le saurons jamais. Mais ce savoir issu de leurs observations et expérimentations a donné assez de résultats pour que, confiantes en elles, elles souhaitent le partager afin que tous les bébés nourris avec la même farine en profitent. Observation, expérimentation, innovation, application d'un savoir, évaluation, partage d'un savoir : tous les éléments clés de l'éthos scientifique sont là!

D'autre part, il y avait les infirmières. Instruites, détentrices d'un savoir issu de la science biomédicale qui leur donnait un statut social particulier, leur rôle social était de transmettre ce savoir, d'y former les «ignorantes », dans le but d'améliorer la santé des populations. Rien, ni dans leur formation ni dans leur statut, ne les préparait à considérer comme valides ou même intéressantes les observations et expérimentations de femmes analphabètes. La réfutation immédiate a fusé, basée sur un argument scientifique qui était, de leur point de vue, implacable. Ont-elles réalisé que cet argument n'avait aucun sens pour les femmes de la coopérative qui, elles, ne voyaient que les bébés et la farine granuleuse et non les «vitamines»? Mon but ici n'est pas de dénoncer la « rigidité cognitive » de l'infirmière ou son éventuel désir d'humilier les femmes de la coopérative qui remettaient en question son savoir (même si ces facteurs ont pu jouer, bien sûr), mais de montrer concrètement comment des

\footnotetext{
${ }^{2}$ Ces exemples sont repris de textes déjà publiés ou sous presse, notamment des extraits d'un chapitre à paraître dans Desgroseilliers, Valérie; Vonarx, Nicolas; Guichard, Anne et Roy, Bernard La santé communautaire dans tous ses états, Québec, PUL (à paraître) et des extraits de Piron, Florence et Thibaut Varin, 2014, «L'affaire Séralini et la confiance dans l'ordre normatif de la science », Implications philosophiques, en ligne à http://www.implications-philosophiques.org/actualite/une/laffaire-seralini-12/.
} 
rapports de pouvoir entre les savoirs sont au cœur des soins de santé, souvent à l'insu des acteurs et actrices du système de santé, et peuvent, en fin de compte, nuire à la santé.

Plus précisément, ce rapport de pouvoir oppose la science biomédicale, hégémonique dans les systèmes de soins de santé dont la qualité est reconnue officiellement par des organismes d'agrément, et ce que je propose d'appeler les savoirs locaux. Ces savoirs locaux débordent de la catégorie bien connue des savoirs traditionnels, propres à une culture non-occidentale, qui définissent à leur manière le corps, la maladie et la santé, ce qu'on appelle aussi parfois les savoirs "endogènes ». Les savoirs locaux incluent aussi les savoirs expérientiels, c'est-à-dire issus de l'expérience vécue ou pratique d'une communauté, qu'elle soit d'ordre cognitif, environnemental, économique ou politique. Par exemple, l'expérience de la pauvreté, de la solitude ou de la pollution crée un savoir de cette expérience chez les personnes qui la vivent et qui en partagent le récit, qui la réfléchissent. Jugés de moindre valeur par la science biomédicale en raison de leur caractère local, situé, donc en principe impossibles à généraliser ou incapables d'accéder à l'universel, ces savoirs locaux sont incompris, voire méprisés ou mis à l'écart par les détenteurs du savoir biomédical, les experts. C'est comme s'il était impossible à ces experts de la science de les comprendre comme étant des « savoirs » : ce sont, pour eux, des «opinions", des «fausses croyances », des mythes, des impressions, des anecdotes, expressions marquant à chaque fois une différence de nature entre ces savoirs et la science. Au mieux, ce sont des «informations » pertinentes. D'ailleurs, en français comme en anglais, le mot « savoir» sonne étrangement au pluriel.

C'est selon moi cette incapacité à considérer que des savoirs issus de non-scientifiques soient valides et intéressants qui empêchent structurellement les scientifiques de contribuer de manière « citoyenne » au débat public sur les sciences et leur impact sur le vivant.

\section{Pouvoir et savoir}

En 2015, le débat médiatique entourant la vaccination a clairement mis au jour cette difficulté des experts de santé publique et de leurs partenaires scientifiques à comprendre la signification des doutes ou des inquiétudes exprimés dans la population à ce sujet. Pour les scientifiques, incluant les journalistes, la seule interprétation possible et pensable de cette situation est que la partie de la population «qui doute», qui questionne les «(bien)faits scientifiques» de la vaccination, est défaillante : douter est ici un signe d'ignorance, d'irrationalité, de stupidité même, à l'encontre en tout cas de la raison, du progrès et même de la santé. Lisons cet extrait d'un article du quotidien Le Soleil, rapportant les propos de Rémi Quirion, scientifique en chef du Québec [4] : "Il est très malheureux de constater que des résultats scientifiques très solides sont ignorés par une certaine fraction de notre population, que ce soit par rapport à la vaccination contre la rougeole ou aux changements climatiques », écrit-il dans une « lettre à la communauté scientifique » envoyée début mars 2015, dans laquelle il dit réfléchir à des moyens pour rétablir la confiance.

«En plus des deux exemples cités par M. Quirion, on pourrait sans doute ajouter des mythes au sujet de la soi-disant toxicité des ondes radio, des OGM et de la fluoration de l'eau ou encore à propos de la soi-disant efficacité de «médecines douces» comme l'homéopathie - mythes qui persistent malgré l'avis d'une écrasante majorité des experts. » [4]

Cette rhétorique est un parfait exemple de la théorie bien connue du « déficit de connaissances » de la population, jugée ignorante et qu'il faut éduquer. Elle oppose la pensée par «mythes » à l'avis des experts qui devrait, pour des personnes rationnelles et raisonnables, être «écrasant» non seulement par sa démultiplication, mais surtout par sa qualité de «scientifique». Beaucoup plus discrètes sont les interprétations de ces doutes qui en font une réaction de prudence critique suscitée par les scandales socio-sanitaires des dernières années, les malversations des compagnies pharmaceutiques, les conflits d'intérêts en recherche biomédicale et les échecs des systèmes de santé à tenir leurs promesses - faits largement rapportés dans les médias. De ce point de vue, les doutes face à la vaccination expriment plutôt un savoir expérientiel qui porte non pas tant sur les vaccins que sur la fiabilité et l'infaillibilité des systèmes de santé - ce qui témoignerait plutôt de l'intelligence d'une population bien informée! 
Cette rhétorique médiatique est étonnante d'un autre point de vue. Elle implique qu'il existe un «avis » scientifique unique sur les vaccins, les OGM, le fluor, les médecines douces, les ondes radio, etc., ce qui lui permet d'opposer en bloc la population inculte et la science écrasante. Or tous les scientifiques savent parfaitement qu'il n'est jamais possible de parvenir à un résultat définitif ou éternel en science, qu'une connaissance scientifique est toujours un modèle, une manière de représenter le réel qui est propre à un moment historique et vouée à se transformer, et que la critique des théories et des manières de produire des faits scientifiques (les méthodes) fait partie intégrante du processus du production des connaissances. Pourtant, lorsque des non-scientifiques tentent de formuler une critique à l'endroit d'une théorie scientifique, cette critique est rapidement reconstruite par les scientifiques comme un «mythe », une croyance irrationnelle persistante et sans fondement qui s'oppose de manière illégitime, voire dangereuse, à la science (au singulier) ou alors comme le symptôme d'une névrose «anti-science» à ne pas prendre au sérieux. Malheureusement, ce type d'argument d'autorité ou de propos méprisant qui cherche à faire taire un débat n'a guère de chance ni de convaincre ni de rétablir la confiance entre la science et la société. En particulier, il ne peut pas vraiment étouffer les soupçons que la science essaie de manipuler l'opinion publique.

\section{Une division cognitive abyssale au cœur de la culture occidentale}

D'où vient cette intolérance de la science aux savoirs locaux sur le vivant? Que signifie-t-elle? Pour le comprendre, je propose d'explorer le cadre normatif de la science, qui domine sa pratique actuelle dans les universités et les centres de recherche. Je voudrais rappeler que ce cadre normatif est construit sur ce que Boaventura de Sousa Santos [5] définit comme une «division abyssale » entre ceux qui savent, les «sachants » comme on dit en France, et ceux qui ne savent pas, qui sont dans l'ignorance et que cette division cognitive fondatrice est au cœur de la culture occidentale depuis le mythe platonicien de la caverne.

En effet, selon ce mythe fondateur de la science (alors appliqué à la philosophie), seuls les philosophes font l'expérience de la connaissance vraie, de la lumière de la connaissance qui rend le monde intelligible, alors que le commun des mortels, enfermé dans la caverne de la vie quotidienne, ne voit que des illusions, des ombres - ce que les sociologues appellent aujourd'hui les préjugés, la doxa ou le sens commun et qui correspondent aux mythes, opinions et croyances évoqués plus haut. L'accès des philosophes à la lumière n'est possible que parce que les non-philosophes sont dans l'obscurité. Ces deux catégories n'existent que parce qu'elles s'opposent. La science a besoin de nonscience pour s'affirmer comme unique savoir de référence.

Bien plus tard, Kant, dans son texte «Qu'est-ce que les Lumières ? (1784) [6], montra qu'il était possible à ces «prisonniers de la caverne » de changer, de passer de leur état de «minorité », de «bétail » incapable de penser sans l'aide d'un directeur de conscience, à l'état de «majorité », c'està-dire à la capacité de penser par eux-mêmes : Sapere aude ! Ose penser par toi-même ! Ose savoir ! Mais ce passage, nous explique Kant, se fait au prix d'un énorme effort personnel et de beaucoup de courage. L'idée que tout le monde peut sortir de «la caverne », de l'ignorance, à condition de travailler fort fut le préalable à une immense démocratisation de l'accès au savoir par le biais de l'éducation pour tous et de son système méritocratique. Les systèmes d'éducation qui sont nés de cette idée restent toutefois structurés autour de la division récurrente entre les sachants (les enseignants) et les ignorants (les élèves) - qui paraît si évidente que personne ne la remet en cause, sauf quelques originaux comme le philosophe des Lumières Joseph Jacotot, maître ignorant convaincu de l'égalité des intelligences [7]). Michel Foucault développa ensuite des analyses percutantes des rapports entre savoir et pouvoir [8] qui ont inspiré tout mon travail sur la science [9].

Le mythe de l'effort et du travail acharné essentiels pour arriver à la connaissance, exemplifié dans la vie et l'œuvre de Kant, ce philosophe si travaillant et rigoureux, persiste dans notre culture. La vie de Marie Curie, cette femme qui a tout donné aux progrès de la connaissance scientifique, y compris la richesse qu'elle aurait pu obtenir si elle avait breveté sa découverte du radium, ainsi que sa santé, a aussi contribué à ce mythe de l'effort, du travail, en somme de la difficulté d'accéder à la connaissance vraie, dans la solitude et l'effort. Pensons aux sacrifices consentis par les doctorants et 
doctorantes pendant plusieurs années, en échange de la reconnaissance éventuelle de la valeur de leur contribution à la science.

Parmi les difficultés à vaincre pour accéder à la connaissance figurent l'acquisition de compétences particulières (cours de méthodes), une bonne connaissance des travaux des « ancêtres » et des maitres du moment (revue de littérature) et surtout des efforts constants pour effacer du travail scientifique les traces des ombres et des illusions qui peuplent le rapport spontané au monde. Ces ombres risquent constamment de ramener dans la caverne la personne qui veut penser et connaitre. L'objectivité ainsi recherchée, qui est un des piliers du cadre normatif dominant de la science, consiste à neutraliser la subjectivité des chercheurs, à les amener à faire abstraction de leur identité sociale, de leurs intérêts et de leurs valeurs, comme s'il s'agissait d'une condition pour vraiment découvrir la réalité telle qu'elle est, dans la lumière de la connaissance. Il est frappant de voir encore de jeunes chercheurs et chercheuses qui sont angoissés à l'idée que leurs activités associatives ou politiques puissent nuire à la crédibilité ou à la légitimité de leur travail parce qu'elles témoigneraient d'une subjectivité encore à l'œuvre ou du moins encore visible. À l'inverse, on pourrait faire valoir que cette expérience leur a ouvert des horizons, a enrichi leur pensée et leur connaissance du monde réel, ce qui leur permettra de produire des connaissances plus fines et plus proches de la complexité de la réalité. Mais le savoir expérientiel des chercheurs eux-mêmes est considéré comme nuisible à la science, si bien que le cadre normatif qui décerne les labels de scientificité leur demande de l'ignorer dans leurs problématiques de recherche et leurs publications scientifiques, générant ainsi une hypocrisie exprimée dans le «nous» de l'écriture scientifique conventionnelle. Les savoirs qui ne respectent pas ce cadre normatif sont exclus du champ scientifique qui revendique le monopole de la vérité, qu'il s'agisse des savoirs expérientiels des informateurs, des savoirs locaux d'une communauté ou de l'expérience humaine et politique des chercheurs. C'est en minorisant ou en ignorant ces savoirs que se construit la légitimité du champ scientifique.

Cette analyse permet de repenser le sentiment d'incompétence (en matière de «savoir») des personnes qui n'ont pas le statut social de «scientifique» et qui sont décrites par les scientifiques comme des «impairs» (comme me l'a dit une fois un chercheur), des «profanes» ou des «citoyens ordinaires »- expressions qui peinent à exprimer de manière positive une catégorie construite sur une exclusion : les non-scientifiques (non-« prêtres », non-citoyens extraordinaires, non-pairs, etc.). Ce sentiment d'incompétence n'est pas lié à la qualité ou la quantité des connaissances des individus, qui peut constamment se modifier, mais à un rapport social qui délégitime les prétentions à la connaissance de ceux et celles qui n'ont pas fait l'effort d'apprendre à être scientifiques, en étudiant de longues années et en obtenant un précieux diplôme de doctorat, par exemple.

Ce sentiment d'incompétence des non-scientifiques, nécessaire à la légitimité du champ scientifique, n'est certainement pas étranger au désintérêt d'une partie de la population pour la science et ses enjeux, ni même, en fin de compte, au rejet des discours scientifiques. La situation paradoxale de la vulgarisation scientifique apparaît : elle doit stimuler l'intérêt des non-scientifiques pour la science tout en maintenant la division cognitive abyssale entre la science et les autres savoirs...

\section{La guerre des savoirs dans le système de santé}

C'est dans ce contexte que la science biomédicale s'est solidement installée comme la référence ultime au sein des systèmes de santé eux-mêmes, articulés autour de la compétence spécifique des docteurs en médecine, les médecins. Alors que ces derniers, dans le fond de leur conscience, connaissent les limites de leur art, savent que leurs diagnostics sont des interprétations dont on peut et doit toujours douter et qu'il faut parfois essayer de nombreuses solutions avant de trouver le remède ou la thérapie qui convient, le discours social leur attribue un contrôle quasi-absolu sur le vivant qui s'ancre dans leur savoir, dans leur maitrise de la science biomédicale acquis au fil de longues études réservées aux « meilleurs ».

Bénéficiant de la légitimité des sachants, la biomédecine a réussi à imposer dans les systèmes de santé une vision universelle des maladies et de leur définition. Classifiées dans des manuels universitaires de diagnostic différentiel totalement imperméables aux savoirs locaux et transmis de 
cohortes en cohortes d'étudiants en médecine à travers le monde, les maladies apparaissent comme des faits scientifiques qu'on "découvre ", alors que le fait même de leur classification est un acte d'interprétation de symptômes et donc une construction théorique. De la même façon qu'il n'y a qu'une seule science, il ne peut y avoir qu'une seule définition officielle des maladies et du corps en bonne santé. Ainsi, considérer le surpoids comme un signe de prospérité et de bonne santé, ce qui est le cas dans de nombreuses sociétés, est, pour la science biomédicale qui a imaginé le "syndrome métabolique », une croyance erronée qui nie le fait qu'il s'agit d'un risque grave pour la santé. La médicalisation des conditions physiques est bien sûr une conséquence de cette emprise de la science sur la santé. Les critiques qui s'inquiètent de la surmédicalisation, par exemple de la grossesse ou de la ménopause, désormais considérées comme des conditions nécessitant de nombreux contrôles médicaux exercés par un médecin, sont vite renvoyées à leur statut de contrevérités anti-science, antiprogrès, anti-santé.

La formation universitaire exclusive des médecins, le recours à la légitimité scientifique pour construire une définition unique, à vocation universelle, des maladies et de la santé et un processus de recherche scientifique rigidifié dans des protocoles hypertechniques, tout ceci contribue à dévaloriser et à exclure du système de santé les savoirs locaux sur la santé et le corps, sur le vivant.

Pourtant, il est possible de sortir de ce cadre normatif et de comprendre que la santé est un idéal qui varie selon les contextes, les époques et les groupes sociaux. On sait bien, par exemple, que toutes les maladies n'inquiètent pas ou ne dérangent pas les individus de la même façon; il y a les optimistes et les hypocondriaques. Mais ce n'est pas qu'une question individuelle, de la même façon que la liberté de conscience ou de choix face au traitement médical n'est pas strictement individuelle. En effet, si un certain état du corps ou de l'âme d'une personne est jugé inacceptable par le groupe social auquel elle appartient, elle sera tentée d'intervenir pour s'auto-normaliser, en particulier en allant «consulter», un conseil qu'elle reçoit souvent. Par exemple, dans notre société obsédée par la performance et la productivité, la distraction et le manque de concentration en classe des enfants sont transformés en une condition (le déficit d'attention) que des parents sont prêts à faire traiter avec un médicament pour éviter l'échec scolaire qui leur est annoncé. L'existence même de cette condition trouve sa légitimité scientifique dans le DSM-5 qui classifie les troubles psychiatriques. Pourtant, d'autres parents se refusent à médicaliser la condition d'enfance, s'inquiétant des effets secondaires des médicaments, mais aussi du choix de résoudre des problèmes humains ou sociaux par la prise d'un médicament [10]. Il suffit de suivre un peu les échanges de groupes d'entraide du TDAH pour découvrir le profond malaise suscité chez les premiers par l'idée que la condition TDAH est une « construction » propre à notre époque et à notre culture, incluant la culture du profit des compagnies pharmaceutiques. Il est toujours possible de juger les parents qui refusent la médication et même la condition TDAH comme étant "anti-science», mais au nom de quoi juge-t-on que leur savoir expérientiel et leur analyse de la situation ne sont pas pertinents, alors qu'il y a débat au sein même de la science sur cette question [11]?

\section{Les scientifiques et la politique}

Il existe actuellement deux grands champs sémantiques associés au terme «politique». D’une part, la politique est associée à quelque chose de sale, de malhonnête, de corrupteur, de « mauvais ». Cela fait référence à l'état de la démocratie contemporaine marquée par un cynisme et un sentiment d'impuissance et de défiance accrus des citoyens face à leurs décideurs, à leurs élites, qui paraissent incapables de résister à la corruption, à la tentation du pouvoir personnel et à la protection de leurs intérêts privés au détriment du bien commun. Dans un contexte néolibéral qui survalorise l'argent et la croissance économique, la confiance dans le politique et dans sa capacité de générer une démocratie inspirant honneur et fierté n'est plus là. Appelons cet état de chose le côté sombre du politique.

En même temps, de nombreux observateurs perçoivent une nouvelle vitalité dans la société civile qui semble vouloir réinventer des formes de vivre-ensemble plus coopératives, plus participatives, plus égalitaires, plus proches de l'idéal du bien commun. Le «politique » peut alors trouver un autre sens comme lieu de débat sur les valeurs et les finalités collectives, d'élaboration de l'action 
commune en vue de construire une Cité juste et accueillante pour tous et de prise de parole individuelle pour faire valoir ce que serait une vie signifiante pour soi et pour autrui. Cette conception éthique du politique (son côté clair) vise une vie bonne, pour et avec autrui, dans des institutions justes; elle apparait bien moins souvent dans les médias que le côté sombre du politique.

Le désir d'agir dans la société exige de prendre en compte les deux côtés du politique. En ignorer le côté sombre maintient la personne dans une situation de naïveté qui, en masquant les obstacles, l'empêche de réaliser ses objectifs ou de participer au débat public. Inversement, se limiter à une version cynique du politique mène à s'auto-exclure de l'aspiration à une vie collective signifiante.

Comme 1'explique Jean-Jacques Salomon dans son grand livre Les scientifiques, entre savoir et pouvoir [12], les scientifiques sont formés à se désintéresser du politique qui leur est toujours présenté comme menaçant et corrupteur. Cette pratique normée de l'apolitisme scientifique les protège peutêtre de certains dérapages, mais elle a au moins deux désavantages majeurs : non seulement elle rend les chercheurs aveugles à la dynamique politique qui anime les rapports entre la science et la société dans laquelle ils travaillent, mais elle les éloigne des débats démocratiques sur les politiques scientifiques qui, pourtant, déterminent les conditions dans lesquelles ils pratiquent la recherche scientifique. Elle limite ainsi considérablement leur capacité d'agir, y compris contre la marchandisation accrue de la connaissance qui se dessine sous leurs yeux par le biais de l'économie du savoir, en complète contradiction avec l'idéal du désintéressement qu'on continue pourtant à leur présenter comme la norme à respecter. C'est le grand déni au cœur du cadre normatif dominant de la science contemporaine.

Cette convention d'apolitisme échoue inévitablement à produire son effet "protecteur » : dès les premières demandes d'admission à un programme de doctorat, les futurs chercheurs non seulement réalisent à quel point la recherche scientifique est profondément politique dans son sens sombre, c'est-à- dire traversée par des passions et des intérêts parfois irrationnels souvent liés à l'argent ou au prestige, mais ils découvrent l'hypocrisie du cadre normatif qui prétend que cela n'existe pas ou ne compte pas. Également coupés du coté clair du politique, non seulement ils ne savent pas comment réintroduire des valeurs et de l'aspiration au bien commun dans leur travail scientifique, mais ils craignent qu'une telle réflexion, par exemple sur la responsabilité sociale de la science, nuise à leur intégration dans la " cité idéale de la science », dans le groupe des pairs.

Cette dimension aussi limite la tenue d'un débat public égalitaire et démocratique sur le vivant dans nos sociétés.

\section{L'ouverture de la science et de la recherche pour permettre le débat public espéré}

Est-il possible de transformer le cadre normatif de la science pour enfin permettre la tenue d'échanges égalitaires et démocratiques, ouverts à toutes les voix, sur le vivant et ce que la science lui fait?

Le mouvement de la science ouverte semble être une voie très féconde en ce sens. Il vise à obtenir aussi bien l'ouverture du processus de recherche aux non-scientifiques et aux experts d'autres disciplines que celle de l'accès aux textes scientifiques (les publications) à ce même public. La multiplication des publics de la science et la participation de citoyens non experts aux processus de recherche pourraient-elles ébranler le syndrome des « sachants » et la fermeture du débat public sur la science?

Le cadre normatif implicite de la science ouverte la conduit à renoncer à la séparation radicale entre la science et la société, au grand partage entre ceux qui savent et ceux qui ne savent pas, au profit d'une justice cognitive reconnaissant et encourageant l'existence d'une pluralité de savoirs complémentaires et non rivaux dans la revendication d'un privilège épistémologique: savoirs scientifiques, savoirs pratiques, savoirs traditionnels, savoirs expérientiels, savoirs politiques. Ce cadre normatif valorise les communautés épistémiques hybrides, formées de chercheurs professionnels et de non-chercheurs, qui se créent actuellement un peu partout dans le monde, dans l'ombre de l'establishment scientifique : la recherche-action collaborative, la science citoyenne (Citizen science), les laboratoires vivants, en somme une multitude de façons de co-créer de la 
connaissance dans un cadre participatif et égalitaire

Mais c'est surtout le libre accès aux articles scientifiques, qu'il se fasse dans les revues scientifiques ou dans les archives ouvertes mises en place par les bibliothèques universitaires, qui joue un rôle majeur dans la transformation contemporaine du cadre normatif de la publication scientifique. Pourquoi? Parce qu'il fait sortir la science des revues scientifiques commerciales payantes où elle était confinée, inaccessible aux non-scientifiques non membres d'une communauté universitaire. Certains critiques estiment que le libre accès ne sert à rien puisque « personne ne lit de science, à part les scientifiques d'un domaine pointu » [13]. Cette approche présuppose le maintien de la division cognitive entre science et non-science et n'envisage pas le «droit collectif à la science » tel que formulé par l'ONU dans un rapport récent qui appuie clairement le libre accès à la science [14].

Un des aspects les plus intéressants de cette science ouverte est l'utilisation des licences Creative Commons à la place du paradigme de la propriété intellectuelle. Une telle licence, accordée par un auteur ou une auteure à son œuvre (article ou livre), autorise sous diverses modalités la libre réutilisation de l'article par ses lecteurs, à condition de toujours mentionner la source. L'utilisation de ces licences est un geste symbolique qui considère la science et ses textes comme un bien commun collectif et non comme un bien privé commercialisable [15].

« Les connaissances sont des ressources sensibles : leur partage permet de réaliser la paix et les autres droits fondamentaux. Au contraire, leur transformation en biens économiques privés dans une " économie de la connaissance » est source d'exclusion, de restriction des savoirs et de limitation de leur circulation. Considérer les connaissances comme des communs ouvre à l'opposé de nouvelles perspectives portées par de nombreux mouvements issus des transformations numériques de la société (logiciels libres, creative commons, accès libre aux publications scientifiques...) » [16].

Il est permis de penser que, dans un tel cadre normatif, la réflexion collective sur les valeurs et la science soit plus inclusive et démocratique.

\section{Références}

1. F. Piron, T. Varin, L'affaire Séralini et la confiance dans l'ordre normatif dominant de la science (1/2), Implic Philos [Internet], 4 juill 2014 [cité 17 janv 2015]; Disponible sur: http://www.implications-philosophiques.org/actualite/une/laffaire-seralini-12/

2. D. Pestre, À contre-science: politiques et savoirs des sociétés contemporaines (Seuil, Paris, 2013).

3. F. Piron, F. Ringtoumda, Les savoirs locaux, la formation non formelle et le développement international: trois études de cas menées au Burkina Faso (Université Laval, Center Sahel, 1994).

4. J.F. Cliche, Connaissances scientifiques : la méfiance du public inquiète les chercheurs, Le Soleil [Internet], 5 mars 2015 [cité 8 mars 2015]; Disponible sur: http://www.lapresse.ca/lesoleil/actualites/science/201503/04/01-4849497-connaissances-scientifiques-la-mefiance-dupublic-inquiete-les-chercheurs.php

5. B. de S. Santos, Beyond Abyssal Thinking: Fron Global Lines to Ecologies of Knowledges, Review, $\mathbf{X X X ( 1 ) ~ ( 2 0 0 7 ) . ~}$

6. E. Kant, Qu'est-ce que les Lumières? [Internet], 1784 [cité 11 juin 2012], Disponible sur: http://fr.wikisource.org/wiki/Qu\%E2\%80\%99est-ce_que_les_Lumi\%C3\%A8res_\%3F

7. J. Rancière, Le maître ignorant: Cinq leçons sur l'émancipation intellectuelle (Poche, Paris, 2004).

8. M. Foucault, Qu'est-ce que les Lumières ? dans Dits et écrits [Internet], (Gallimard, Paris, 1984) [cité 10 juill 2013]. Disponible sur: http://1libertaire.free.fr/Foucault17.html 
9. F. Piron, Savoir, pouvoir et éthique de la recherche, dans A. Beaulieu, (éd.), Michel Foucault et le contrôle social [Internet] (Presses de l'Université Laval, Québec 2005) [cité 28 mai 2015]. p. 130 50. Disponible sur: https://halshs.archives-ouvertes.fr/halshs-00806359

10. J-C. St-Onge, TDAH?: pour en finir avec le dopage des enfants (Écosociété, Montréal, 2015).

11. M-P Genecand, Et si l'hyperactivité n'existait pas? Le Temps [Internet], (Genève, 24 févr 2015) [cité 17 mars 2015]; Disponible sur: http://www.letemps.ch/Page/Uuid/56b6eb8c-bb83-11e4b1aa-59105399a835/Et_si_lhyperactivité_nexistait_pas

12. J-J. Salomon, Les Scientifiques : Entre pouvoir et savoir (Albin Michel, Paris, 2006).

13. F. Piron, P. Lasou, Pratiques de publications, dépôt institutionnel et perception du libre accès. Enquête auprès des chercheuses et chercheurs de l'Université Laval, [Internet], (Université Laval, Québec, 2014 p. 92. Disponible sur:

http://www.bibl.ulaval.ca/fichiers_site/services/libre_acces/pratiques-de-publication-libreacces.pdf

14. F. Shaheed, Politiques en matière de droit d'auteur et droit à la science et à la culture [Internet], (Organisation des Nations Unies, Genève, 2015 mars [cité 15 mars 2015]. Report No.:

A/HRC/28/57. Disponible sur: http://www.ohchr.org/EN/HRBodies/HRC/RegularSessions/Session28/Documents/A_HRC_28_ 57_FRE.doc

15. S. Couture, Science et bien commun : les transformations des pratiques scientifiques à l'ère du numérique - Ritimo [Internet], (2014) [cité 16 mars 2015]. Disponible sur: http://www.ritimo.org/article5400.html

16. H. Le Crosnier, En communs. Une introduction aux communs de la connaissance (C \& $\mathrm{F}$ Éditions, Caen, 2015). 
